\title{
Vanishing Waves on Semi-closed Space Intervals and Applications in Mathematical Physics
}

\author{
Ghiocel Toma
}

Department of Applied Sciences, Politehnica University, Bucharest, Romania

\begin{abstract}
Test-functions (which differ to zero only on a limited interval and have continuous derivatives of any order on the whole real axis) are widely used in the mathematical theory. Yet less attention was given to intuitive aspects on dynamics of such test functions or of similar functions considered as possible solution of certain equations in mathematical physics (as wave equation). This study will show that the use of wave equation on small space interval considered around the point of space where the sources of the generated field are situated can be mathematically represented by vanishing waves corresponding to a superposition of travelling test functions. As an important consequence, some directions for propagating the generated wave appears and the possibility of reverse radiation being rejected. Specific applications for other phenomena involving wave generation (as the Lorentz formulae describing the generation of a wave with different features after the interaction with the observer's material medium are also presented.
\end{abstract}

Keywords: vanishing waves, test functions, semiclosed intervals.

\section{Introduction}

Test-functions (which differ to zero only on a limited interval and have continuous derivatives of any order on the whole real axis) are widely used in the mathematical theory of distributions and in Fourier analysis of wavelets. Yet such test functions, similar to the Dirac functions, can't be generated by a differential equation. The existence of such an equation of evolution, beginning to act at an initial moment of time, would imply the necessity for a derivative of certain order to make a jump at this initial moment of time from the zero value to a nonzero value. But this aspect is in contradiction with the property of test-functions to have continuous derivatives of any order on the whole real axis, represented in this case by the time axis. So it results that an ideal test-function can't be generated by a differential equation (see also [1]); the analysis has to be restricted at possibilities of generating practical test-functions (functions similar to test-functions, but having a finite number of continuous derivatives on the whole real axis) useful for wavelets analysis. Due to the exact form of the derivatives of test-functions, we can't apply derivative free algorithms [2] or algorithms which can change in time [3. Starting from the exact mathematical expressions 
of a certain test-function and of its derivatives, we must use specific differential equations for generating such practical test-functions.

This aspect is connected with causal aspects of generating apparently acausal pulses as solutions of the wave equation, presented in 44. Thus, such testfunctions, considered at the macroscopic scale (that means not as Dirac-functions) can represent solutions for certain equations in mathematical physics (an example being the wave-equation). The main consequence of this consists in the possibility of certain pulses to appear as solutions of the wave-equation under initial null conditions for the function and for all its derivatives and without any free-term (a source-term) to exist. In order to prove the possibility of appearing acausal pulses as solutions of the wave-equation (not determined by the initial conditions or by some external forces) we begin by writing the wave-equation

$$
\frac{\partial^{2} \phi}{\partial x^{2}}-\frac{1}{v^{2}} \frac{\partial^{2} \phi}{\partial t^{2}}=0
$$

for a free string defined on the length interval $(0, l)$ (an open set), where $\phi$ represents the amplitude of the string oscillations and $v$ represents the velocity of the waves inside the string medium. At the initial moment of time (the zero moment) the amplitude $\phi$ together with all its derivatives of first and second order are equal to zero. From the mathematical theory of the wave-equation we know that any solution of this equation must be a superposition of a direct wave and of a reverse wave. We shall restrict our analyze at direct waves and consider a supposed extension of the string on the whole Ox axis, $\phi$ being defined by the function

$$
\phi(\tau)=\left\{\begin{array}{r}
\exp \left(\frac{1}{(x-v t+1)^{2}-1}\right) \text { for }|x-v t+1|<1 \\
0 \text { for }|x-v t+1| \geq 1
\end{array}\right.
$$

where $t \geq 0$. This function for the extended string satisfies the wave-equation (being a function of $\mathrm{x}-\mathrm{vt}$, a direct wave). It is a continuous function, having continuous partial derivatives of any order for $x \in(-\infty, \infty)$ and for $x \geq 0$. For $x \in(0, l)$ (the real string)the amplitude $\phi$ and all its derivatives are equal to zero at the zero moment of time, as required by the initial null conditions for the real string (nonzero values appearing only for $x \in(-2,0)$ for $t=0$, while on this interval $|x-v t+1|=|x+1|<1)$. We can notice that for $t=0$ the amplitude $\phi$ and its partial derivatives differ to zero only on a finite space interval, this being a property of the functions defined on a compact set (test functions). But the argument of the exponential function is $x-v t$; this implies that the positive amplitude existing on the length interval $(-2,0)$ at the zero moment of time will move along the $\mathrm{Ox}$ axis in the direction $x=+\infty$. So at some time moments $t_{1}<t_{2}<t_{3}<t_{4}<\ldots$ after the zero moment the amplitude $\phi$ will be present inside the string, moving from one edge to the other. It can be noticed that the pulse passes through the real string and at a certain time moment $t_{\text {fin }}$ (when the pulse existing at the zero moment of time on the length interval $(-2,0)$ has moved into the length interval $(l, l+2))$ its action upon the real string ceases. We must point the fact that the limit points $x=0$ and $x=l$ are not considered 
to belong to the string; but this is in accordance with the rigorous definition of derivatives (for these limit points can't be defined derivatives as related to any direction around them).

This point of space (the limit of the open space interval considered) is very important for our analysis, while we shall extend the study to closed space intervals. Considering small space intervals around the points of space where the sources of the generated field are situated (for example, the case of electrical charges generating the electromagnetic field), it will be shown that causal aspects require the logical existence of a certain causal chain for transmitting interaction from one point of space to another, which can be represented by mathematical functions which vanishes (its amplitude and all its derivatives) in certain points of space. From this point of space, an informational connection for transmitting the wave further could be considered (instead of a tranmission based on certain derivatives of the wave). Thus a kind of granular aspect for propagation along a certain axis can be noticed, suitable for application in quantum theory. As an important consequence, by a multiscale analysis and the use of non-Markov systems, some directions for propagating the generated wave will appear and the possibility of reverse radiation will be rejected. Finally. specific applications for other phenomena involving wave generation (as the Lorentz formulae describing the generation of a wave with different features after the interaction with the observer's material medium) will be also presented.

\section{Test-Functions for Semi-closed Space Intervals}

If we extend our analysis to closed intervals by adding the limit of the space interval to the previously studied open intervals (for example by adding the points $x=0$ and $x=l$ to the open interval $(0, l)$, we should take into account the fact that a complete mathematical analysis usually implies the use of a certain function $f(t)$ defined at the limit of the working space interval (the point of space $x=0$, in the previous example). Other complete mathematical problems for the wave equation or for similar equations in mathematical physics use functions $f_{0}(t), f_{l}(t)$ corresponding to both limits of the working space intervals (the points of space $x=0$ and $x=l$ in the previous example) or other supplementary functions.

The use of such supplementary functions defined on the limit of the closed interval could appear as a possible explanation for the problem of generating acausal pulses as solutions of the wave equation on open intervals. The acausal pulse presented in the previous paragraph (similar to wavelets) travelling along the $\mathrm{Ox}$ axis requires a certain non-zero function of time $f_{0}(t)$ for the amplitude of the pulse for the limit of the interval $x=0$. It could be argued that the complete mathematical problem of generating acausal pulses for null initial conditions on an open interval and for null functions $f_{0}(t)$ and $f_{l}(t)$ corresponding to function $\phi$ (the pulse amplitude) at the limits of the interval $x=0$ and $x=l$ respectively, would reject the possibility of appearing the acausal pulse presented in the previous paragraph. The acausal pulse $\phi$ presented implies non-zero values 
for $f_{0}$ and $f_{l}$ at the limit of the closed interval at certain time moments, which represents a contradiction with the requirement for these functions $f_{0}$ and $f_{l}$ to present null values at any time moment. By an intuitive approach, null external sources would imply null values for functions $f_{0}$ and $f_{l}$ and (as a consequence) null values for the pulse amplitude $\phi$.

Yet it can be easily shown that the problem of generating acausal pulses on semi-closed intervals can not be rejected by using supplementary requirements for certain functions $f(t)$ defined at one limit of such space intervals. Let us simply suppose that instead of function

$$
\phi(\tau)=\left\{\begin{array}{r}
\exp \left(\frac{1}{(x-v t+1)^{2}-1}\right) \text { for }|x-v t+1|<1 \\
0 \text { for }|x-v t+1| \geq 1
\end{array}\right.
$$

presented in previous paragraph we must take into consideration two functions $\phi_{0}$ and $\phi_{l}$ defined as

$$
\phi_{0}(\tau)=\left\{\begin{aligned}
\exp \left(\frac{1}{(x-v t+m)^{2}-1}\right) & \text { for }|x-v t+1|<1 \\
0 & \text { for }|x-v t+m| \geq 1
\end{aligned}\right.
$$

and

$$
\phi_{l}(\tau)=\left\{\begin{array}{r}
-\exp \left(\frac{1}{(x+v t-m)^{2}-1}\right) \text { for }|x-v t+1|<1 \\
0 \text { for }|x+v t-m| \geq 1
\end{array}\right.
$$

with $m$ selected as $m>0, m n-1>l$ (so as both functions $\phi_{0}$ and $\phi_{l}$ to have non-zero values outside the real string and to be asymmetrical as related to the point of space $x=0$. While function $\phi_{0}$ corresponds to a direct wave (its argument being $(x-v t)$ ) and $\phi_{l}$ corresponds to a reverse wave (its argument being $(x+v t))$ it results that both functions $\phi_{0}$ and $\phi_{l}$ arrive at the same time at the space origin $x=0$, the sum of these two external pulses being null all the time (functions $\phi_{0}$ and $\phi_{l}$ being asymmetrical, $\phi_{0}=-\phi_{l}$ ). So by requiring that $\phi(t)=0$ for $x=0$ (the left limit of a semi-closed interval $[0, l)$ ) we can not reject the mathematical possibility of appearing an acausal pulse on a semiclosed interval.

This pulse is in fact a travelling wave propagating from $x=-\infty$ towards $x=\infty$ which vanishes at the point of space $x=0$. Moreover, its derivatives are also equal to zero at this point of space for certain time moments (when both travelling pulses cease their action in the point of space $x=0$ ). This pulse is a solution of the wave-equation on the semi-closed interval $[0, l)$, and can be very useful for considering a transmission of interaction on finite space-intervals (in our case the interaction being transmitted from $x=l$ towards $x=0$ ). From this point of space, at the time moments when the amplitude and all its derivatives are equal to zero the interaction can be further transmitted by considering an informational connection; the mathematical form of the pulse is changed, a new wave should be generated for the adjacent space interval and a mathematical connection for transmitting further the interaction (towards $x=-\infty)$ is not possible while the pulse amplitude and all its derivatives vanish at this time moments at the point of space $x=0$. This aspect implies a step-by 
step transmission of interaction starting from an initial semi-closed interval (its open limit corresponding to the source of the fieldd, for example) to other space intervals. This corresponds to a granular aspect of space suitable for applications in quantum physics, where the generation and annihilation of quantum particles should be considered on limited space-time intervals. For this purpose, specific computer algorithms and memory cells corresponding to each space interval should be used. The informational connection from one space interval to another should be represented by computer operations of data initialization.

\section{Aspects Connected with Spherical Waves}

A possible mathematical explanation for this aspect consists in the fact that we have used a reverse wave (an acausal pulse) propagating from $x=\infty$ towards $x=-\infty$, which is first received at the right limit $x=l$ of the semi-closed interval $[0, l)$ before arriving at the point of space $x=0$. It can be argued that in case of a closed space interval $[0, l]$ we should consider the complete mathematical problem, consisting of two functions $f_{0}(t), f_{l}(t)$ corresponding to both limits of the working space intervals (the points of space $x=0$ and $x=l$. But in fact the wave equation corresponds to a physical model valid in the three-dimensional space, under the form

$$
\frac{\partial^{2} \phi}{\partial x^{2}}+\frac{\partial^{2} \phi}{\partial y^{2}}+\frac{\partial^{2} \phi}{\partial z^{2}}-\frac{1}{v^{2}} \frac{\partial^{2} \phi}{\partial t^{2}}=0
$$

and the one-dimensional model previously used is just an approximation. Moreover, the source of the field is considered at a microscopic scale (quantum particles like electrons for the case of the electromagnetic field, for example) and the emitted field for such elementary particles presents a spherical symmetry. Transforming the previous equation in polar coordinates and supposing that the function $\phi$ depends only on $r$ (the distance from the source of the field to the point of space where this emitted field is received), it results

$$
\frac{\partial^{2} U}{\partial r^{2}}-\frac{1}{v^{2}} \frac{\partial^{2} U}{\partial t^{2}}=0
$$

where

$$
U=r \varphi
$$

An analysis of the field emitted from the space origim towards a point of space $r=r_{0}$ (where the field is received) should be performed on the space interval $(0, r]$ (a semi-closed interval); the point of space $r=0$ can not be included in the working interval as long as the solution $\phi(r)$ for the field is obtained by dividing the solution $U(r)$ of the previous equation (in spherical coordinates) through $r$ (the denominator of the solution $\phi$ being zero, some supplementary aspects connected to the limit of functions should be added, but still without considering a solution for the space origin). 
Thus an asymmetry in the required methods for analyzing phenomena appears. In a logical manner, by taking also into consideration the free-term (corresponding to the source of the field) situated in the point of space $x=0$ (the origin) it results that the use of function depending on $x-v t$ (mentioned in the previous paragraph) or $r-v t$ (for the spherical waves) represents also a limit for the case of a sequence of small interactions acting as external source (freeterm)- changes in the value of partial derivatives as related to space coordinates - changes in the partial derivatives of the amplitude as related to time - changes in the value of the function, so as the possibility of appearing acausal pulses (not yet observed) to be rejected. Such a causal chain can be represented in a mathematical form as a differential equation able to generate functions similar to test functions, defined as practical test functions only as an approximation at a greater scale of space-time for the case when the length and time intervals corresponding to such equations with finite differences are very small. Moreover, a certain direction for the transmission of interaction appearing, it results that the possibility of reverse radiation (a reverse wave generated by points of space where a direct wave has arrived) should be rejected in a logical manner (a memory of previous phenomena determining the direction of propagation). Mathematically, an analysis at a small spatial and temporal scale based on continuous functions for transmitting interactions from one point of space to another (similar to continuous wave function in quantum physics describing the generation and annihilation of elementary particles) should be described by non-Markov processes (phenomena which should be analyzed by taking into account the evolution in a past time interval).

\section{Applications at Relativistic Transformation of Waves}

An application of such non-Markov processes should be the analysis of Lorentz transformation in special relativity, when a certain wave-train interacts with the observer's material medium. The usual interpretation of special relativity theory considers that the Lorentz formulae describe the transformation of the space-time coordinates corresponding to an event when the inertial reference system is changed. These formulae are considered to be valid at any moment of time after a certain synchronization moment (the zero moment) irrespective to the measuring method used. However, there are some problems connected to the use of mechanical measurements on closed-loop trajectories with analysis performed on adjoined small time intervals. For example, if we consider that at the zero moment of time, in a medium with a gravitational field which can be neglected, two observers are beginning a movement from the same point of space, in opposite directions, on circular trajectories having a very great radius of curvature, so as to meet again after a certain time interval, we can consider the end of each small time interval as a resynchronization moment and it results that time dilation appears on each small time interval. Yet if we consider that the time intervals measured after a resynchronization procedure can be added to the previously measured time intervals (the result being considered as related to 
the initial time moment) a global time dilation appears. If the time is measured using the age of two plates, it results that the plate in a reference system $S_{2}$ is older than the other in reference system $S_{1}$, (having a less mechanical resistance) and it can be destroyed by it after both observers stop their circular movements. However, the same analysis can be made by starting from another set of small time intervals considered in the other reference system, and finally results that the plate in reference system $S_{1}$ is older than the other in reference system $S_{2}$, (having a less mechanical resistance) and it can be destroyed by it after both observers stop their circular movements. But this result is in logic contradiction with the previous conclusion, because a plate can not destroy and in the same time be destroyed by another one.

A logical attempt for solving this contradiction can be made by considering that Lorentz formulae are valid only for electromagnetic phenomena (as in the case of the transversal Doppler effect) and not in case of mechanical phenomena or any phenomena involving memory of previous measurements. Using an intuitive approach which considers that the Lorentz transformation represents physical transformation of a wave-train when this interacts with the observer's material medium, such logical contradiction can be avoided (see [5], 6] for more details). Yet the memory of past events can not be totally neglected. The transformation of a received wave into another wave which moves along the same direction, with certain mathematical expressions describing how space-time coordinates corresponding to the case when the received wave would have been not affected by interaction are transformed into space-time coordinates corresponding to the transformed wave train (according to the Lorentz formulae valid on the space and time intervals corresponding to the received wave-train) requires a certain memory for the received wave train necessary for performing the transformation (other way no local time dilation would appear). This aspects is similar to the requirement of using non-Markov processes for justifying how a certain direction of propagation for the generated wave appears.

\section{Conclusions}

This study has shown that some solutions of the wave equation for semi-closed space interval considered around the point of space where the sources of the generated field are situated (for example, the case of electrical charges generating the electromagnetic field) can be mathematically represented by vanishing waves corresponding to a superposition of travelling test functions. It is also shown that this aspect requires the logical existence of a certain causal chain for transmitting interaction from one point of space to another. As an important consequence, by a multiscale analysis and the use of non-Markov systems, certain directions for propagating the generated wave appeared and the possibility of reverse radiation was rejected. Specific applications for other phenomena involving wave generation (as the Lorentz formulae describing the generation of a wave with different features after the interaction with the observer's material medium) have been also presented. Unlike other mathematical problems (Cauchy problem) based on 
long-range dependence (see also [7], where statistical aspects are also taken into consideration), this study presents aspects connected to short-range interactions. Asymptotic properties are taken into account for the mathematical problem for functions having the limit $\infty$ (null denominator at the limit of the working semiclosed interval) instead of an approach based on relaxation phenomena, as in [8]. In future studies, such aspects will be extended to mathematical models describing step changes in a certain environment (similar to aspects presented in [9], [10] with step changes presented in [11. The aspects presented in this study can be extended at closed space intervals, by considering that at the initial moment of time, at one of the spatial limits of the interval arrives a direct wave and a reverse wave (asymmetrical as related to this point of space) represented both by sequences of extended Dirac pulses having the same spacelemgth $d=L / k(k$ being an integer and $L$ being the length of the whole interval). As a consequence, after a certain time interval, a set of oscillations represented by stationary waves with null derivatives of certain orders at both spatial limits will apeear.

Acknowledgment. This work was supported by the National Commission of Romania for UNESCO, through a pilot grant of international research involving Politehnica University, Salerno University, IBM India Labs and Shanghai University.

\section{References}

1. Toma, C. : Acausal pulses in physics-numerical simulations, Bulgarian Journal of Physics (to appear)

2. Morgado, J. M., Gomes, D.J. : A derivative - free tracking algorithm for implicit curves with singularities, Lecture Notes in Computer Science 3039 (2004) 221-229

3. Federl, P., Prudinkiewiez, P. : Solving differential equations in developmental models of multicellular structures using L-systems, Lecture Notes in Computer Science 3037 (2004) 65-82

4. Toma, C.: The possibility of appearing acausal pulses as solutions of the wave equation, The Hyperion Scientific Journal 41 (2004), 25-28

5. Toma, C.: A connection between special relativity and quantum theory based on non-commutative properties and system - wave interaction, Balkan Physics Letters Supplement 5 (1997), 2509-2513

6. Toma, C.: The advantages of presenting special relativity using modern concepts, Balkan Physics Letters Supplement 5 (1997), 2334-2337

7. Li, M., Lim, S.C.: Modelling Network Traffic Using Cauchy Correlation Model with Long-Range Dependence, Modern Physics Letters,B 19 (2005), 829-840

8. Lim, S.C., Li, M. : Generalized Cauchy Process and Its Application to Relaxation Phenomena, Journal of Physics A Mathematical and General 39 (2004), 2935-2951

9. Lide, F., Jinhai, L., Suosheng C.: Application of VBA in HP3470A Data Acquisition System, Journal of Instrunebtation and Measurements 8 (2005), 377-379

10. Lide, F., Wanling, Z., Jinha, L., Amin, J.: A New Intelligent Dynamic Heat Meter, IEEE Proceedings of ISDA 2006 Conference, 187-191

11. Xiaoting, L., Lide, F.: Study on Dynamic Heat Measurement Method after Step Change of System Flux, IEEE Proceedings of ISDA 2006 Conference, 192-197 